\title{
Green Computing using Multi label classification for Mobile Medical Recommendation
}

\author{
T Sivakumar ${ }^{1}$, Basheer $\mathbf{P}^{2}$, Mubeena $\mathbf{V}^{3}$ \\ Assistant Professor, Dept of Computer Science, Sree Narayana Guru College, K. G. Chavadi, Coimbatore, Tamil Nadu ${ }^{1}$ \\ M. Phil Scholar, Dept of Computer Science, Sree Narayana Guru College, K. G. Chavadi, Coimbatore, Tamil Nadu ${ }^{2,3}$
}

\begin{abstract}
Communication technology plays a major role for sharing the knowledge with the support of customer friendly service. Customer friendly technology like cellular helps to exchange the knowledge between people. However, such services require more computing resources and energy. For energy consumption green computing were used to balance the problem. Henceforth development of green and energy-efficient in cellular application has become an important topic in communication technology. For this analyses this paper propose a multi label classification methods for medical recommendation system through mobile application. Using multi label classification method we labelled it on doctor recommendation system and this improve to provide needed data to the user.
\end{abstract}

Keywords: Green computing, Data mining, Classification and Medical recommendation.

\section{I.INTRODUCTION}

Data Mining (DM) is the adept of tenacity expensive association i.e. diminishes the power bill.Uses of noninformation from the huge database. The persistence of the toxic material in the equipment's make the employee safe DM is to discover information and contemporaneous it in from health difficult and professional hazards [3]. In the an understanding that is simply comprehensible to the extensive term these green equipment will be less costly people. Knowledge recognition in database is exhaustive deprived of any hidden cost of surplus and improved method which offering a number of functional, relevant reserve consumption without any detrimental effect of information [1]. On the other hand energy efficient is an exactness, performance and durability. However, the imperative factor, energy competentGreen computing can boundaries of data rates and resources significantly disturb be distinct as the efficient use of computing possessions. It the user involvement. Label-based methods, such as label is the name devoted to the movement which characterizes position and label classification, play significant roles in an ecologically responsible way of calculating through mobile recommendation systems. This paper emphasis on reduced power ingesting. It is also connected with the Multi Label Classification (MLC) for cracking this issues. proper use of computing resources and plays a major role MLC is a variant of the classification problem in which in diminishing their hazardous impact on background. numerous target labels must be allocated to each instance. Two main issues concomitant with green computing are: This method has been generally employed in many decrease in energy consumption and pollution control. research areas. With the help of the extrapolative analyses While the previous can be achieved by proper use of classification can done easily. Predictive analytics is used electronic good and through advance of energy efficient to habituallyanalyse large amounts of data with unequal and less power overwhelming hardware, the later can be variables. The most ordinary and important applications in accomplished through their compact use, proper data mining virtually certainly conquer predictive reprocessing policies and use of fewer toxic elements in modelling [4].

developed the equipment's. Exploiting economic Classification refers to the prediction of a target unreliable feasibility and ensuring sustainability are between the that is uncompromising in nature, such as deduction other characteristics of green computing [2]. Out of these healthcare racket. Presumption, on the other hand, refers to above specified aspects of green computing, in this paper, the calculation of a objective variable that is metric (i.e., we are converging on mobile application for interval or ratio) in scenery, such as predicting the degree recommendation systems. The development of green of stay or the quantity of resource utilization. For computing and energy-efficient $5 \mathrm{G}$ submissions has predictive illustration the data mining techniques convert one of the core topics in communications. Bearing frequently used encompass traditional statistics, such as in mind the heavy petition for this field, unconventional numerous distinguish analysis and logistic deterioration mobile applications with high-performance algorithms investigation [5].In real life, the aforementioned attract the consideration of researchers. Recommendation assumption is not applicable to many of the more systems are commonly used to predict the "rating" or complicated questions of machine learning. One primary "preference", that a user would give to an item.Green reason is that the samples from real life are extremely computing diminishes the energy consumption of the complicated, and one sample can simultaneously contain 
several pieces of semantic information. To overcome this real-life issue regarding how one sample could contain multiple pieces of semantic information, one straightforward method is to assign an appropriate label set for one sample to represent its semantics. This type of classification problem of models is called multi-label classification. In contrast to traditional classification, in multi-label classification, one sample is represented by an eigenvector and a label set rather than by one label exclusively. The task of multi-label classification is to train a function to forecast the unknown sample and return a label set. The formal definition of multi-label classification is asfollows: assume that $\mathrm{A}=\mathrm{R}^{\mathrm{m}}$ is an $\mathrm{m}$ dimensional eigenvector space and that $B=\{b 1, \ldots$, bd \} is the label space containing $d$ labels. The specific task of multi-label classification is to learn a function, $\mathrm{h}: \mathrm{A} \rightarrow 2^{\mathrm{B}}$ , from the training dataset $\mathrm{D}=\{(\mathrm{ai}, \mathrm{bi})\}_{\mathrm{i}=1}^{\mathrm{n}}$. For each multi-label sample $\left(a_{i}, b_{i}\right), a_{i}$ is an m-dimensional eigenvector, and $b_{i}$ is a label set connected to $a_{i}$ (denoted by a k-dimensional vector; namely, the label set contains $\mathrm{d}$ labels). For each unknown sample a $\in$ A, the multi-label classifier $\mathrm{h}(\cdot)$ forecasts an appropriate label set $\mathrm{h}(\mathrm{a}) \subseteq \mathrm{B}$. Mainly multi-label classification primarily focused on the multi-label classification problem of text. Over the past ten years, multi-classification has gradually received attention from the machine learning community and other relevant fields and has been broadly applied to various areas, ranging from the denotation of multimedia content to fields of biological information, webpage mining, rule mining, information indexing, and label recommendation.

In recommendation applications, such as text classification, internet advertising, and music classification, the number of labels is generally tens of thousands to hundreds of thousands, and this number is still growing [6]. Therefore, it is important to propose an efficient method for accomplishing these tasks. In multilabel classification, because each sample can be assigned multiple labels, the task becomes challenging. To solve this issue this paper proposes a data mining classification method for recommendation through mobile application by using the medical dataset. To classify the user required data through mobile application we use K-medoids algorithm to find out the nearest element in the labelled data and also feature extraction to find out the most likelihood data.

\section{LITERATURE REVIEW}

Bernhard Peischl et al [7], reports on collaborative work with an SME, emergent a system for data procurement in health care associations, providing mobile data funding. We fleetingly introduce the ICF and the ICD classification system from the WHO as a substance for our mobile application. A two-staged usability calculation in a very early stage of development allows us to participate usercentred design in the mobile application development process. Our practice comprises consultations and usability tests with aimperfect number of users and thus can even be achieved within a resource-constrained setting as it is naturally found in smaller software advance teams. We discuss the associated results of the usability tests quantitatively and qualitatively. Since these outcomes we deduce recommendations (and open issues) regarding the user boundary design of the mobile application.

SunitaSoni and Ranjita Panda [2,8]analyse the Rapid development and heavy uses of electronic gadgets through the last two periods has led to generation of a huge quantity of electronic wastes resulting in soil, water and environmental pollutions. Thus pollution control and environmental security has develop the greatest concern of conservational scientists and activists worldwide. Clearance of electronic wastes, one of the consequences of this suburbanisation process has converted a major difficult in our society. Since these wastes are not decomposable, gradual admission of these e-wastes leads to accumulation of various toxic metals like lead $(\mathrm{Pb})$, cadmium $(\mathrm{Cd})$ etc. and pollutes the soil and the ground water. Ground water pollution in turn, affects the plant inborn and the living scheme as a whole causing severe health dangers and disorders. Therefore, proper organization of these electronic wastes has become aunrelenting demand of the time. In this paper, we converse about countless sources of e-wastes, their possessions and recommend steps for supervision of these toxic and unsafe wastes so as to make the advance process supportable and green.

PranaliDhongade et al[9] analyse the modern applications compulsory multi-label classification such as protein utility classification, music categorization, gene function investigation and semantic division classification. Multilabel learning trainings the problem wherever each instance related with a set of labels instantaneously. There are many conducts to solve the difficult of multi-label classification. The basic approach to solve the difficult is to label the data, but it has been found that progression of labelling to multi-label data is expensive and time overriding. Problem transformation and Algorithm variation are the two methods of multi-label classification. In problem conversion methods, convert multi-label problem into set of binary cataloguingunruly which can then be touched using single-class classifiers and algorithm variation methods are those procedures that extend detailed learning algorithms in order to switch multi-label data directly. Transductive based multi-label classification is an operative way of assigning multi-label to each occurrence. TRAM algorithm used label set process which utilize the material of label and unlabeled data which supports to adjust the problem of amalgamatedlabeling.

Shubpreet Kaur and Dr.R.K.Bawa [10] summarize various mechanical articles on medical diagnosis and prediction.It has also been compensating attention on existingexplore being approved out using the data mining process to expand the disease(s) calculating procedure. They present upcoming leanings of current procedures of KDD, using data mining tools for healthcare. It also present imperative 
issues and encounters connected with data mining and healthcare in mutual. The investigate found a growing number of data mining submission counting psychotherapy of health care borderline for superior metier policy-making, uncovering of sickness occurrence and pointless hospital deaths. The root foundation of all diseases get quicker in the direction of drugs i.e. the primary risk factor of all side-splitting sicknesses. Drug requirement using WEKA has been used that transport into light regardingmultitude of drug abusers in growthmistreating drugs at age below 20yrs.It is to hypothesis attentive the druggist about the different sicknesses that are caused with important or long term incorporation of drugs in their life.

DivyaTomar and Sonali Agarwal [11] in their survey explore the helpfulness of various Data Mining systems such as classification, clustering, association, waning in health domain. They contemporary a brief introduction of these method and their compensations and difficulties. This review also highlights submissions, confront and outlook issues of Data Mining in healthcare. For successful ingesting of data mining in health organizations there is a need of enlarge and secure health data circulation among unlike get-togethers. Some good manners limitations such as contractual associations between scientist and health care society are required to trounce the sanctuary issues. There is also a need of similar approach for generating the data warehouse.

S. DivyaMeena and M. Revathi, et al [12] Healthcare is positively a substantial pointer for the development of society. Health does not only represent as dearth of disease but also potential to take in for inquisitive one's probable. In actuality, there is a big discontinuity among the pastoral and urban health tune capability and handiness. They diagnose some of the misfortunes in Indian healthcare and challenge to make available a description by notice the potential of healthcare. So, the amenities cause to be by healthcare are not a mere responsibility of medical grassland but also of information knowledge. In fact, data mining plays abouncing role in have enough money a trustworthyexactness in guess the diseases and its jeopardy factors. Some of the data mining suggestion and practises used in real world are opposingContextual of a Predictive Analytics Tool, its area and the method to compute the number of days a patient is probable to be divulge to hospital.

\section{RECOMMENDATION SYSTEM USING PREDICTIVE}

With the support of the DM analytical analyses it mines the possible result. Predictive analytics is used to conclude the possible outlook result of an experience or the likelihood of a conditions happening. Predictive model of $\mathrm{DM}$ is very essential for the fitness sector to analyse the disease. The exposed knowledge can be used by the healthcare administrator to progress the dominance of service. In healthcare, data mining is attractive gradually more well-liked, if not increasingly more indispensable. Several factors have provoked the use of data mining applications in healthcare. Our proposed methods to foresee labels conforming to doctor knowledge. The tags can then be used to matchpatients and doctors in the recommendation scheme.

\section{DATA PRE-PROCESSING}

The data processing mission is also one of the principles which must be occupied care in the procedure of data mining. The data contribution to a data mining algorithm necessity not be in goodformat and is hence not apposite for processing efficiently. In such a case, we essential to see the data is in proper arrangement so that it is appropriate for processing. This case commonly arrives when we try to mine the data using the prevailing data mining tools or processes [13]. Different Data mining tools accessible in the market have changed formats for input which makes the user forced to transform the prevailing input dataset into the new format. This situation is very time overriding, laborious and has a coincidental of data loss as the data is to be pass in automatically into a new format to be maintained by the tool. The original doctor information necessitates pre-processing. Each doctor has a conforming feature route $\mathrm{x}$ and a label vector $y$. We picked anassembly of doctors haphazardly and label each doctor with the situations with which they are most skilled. After the labeling, we have d dissimilar labels in total. The label vector container then be characterized as a d-dimensional vector. Each dimension of the vector characterises whether adoctor is accomplished in treating a particular condition. If a doctor isskilled in giving a specific condition, then the conformingvalue in the vector is set to 1 ; otherwise, it is set to 0 .

\section{EXTRACTING FEATURES FOR DOCTOR RECOMMENDATION}

The processing of the feature extraction is more complex.We report three types of features, which are explained asfollows. (i) Classification features include evidence suchas the hospital name, department, title, or partner. Such structures must be encoded. For eg, there are ci conceivablevalues of doctor titles in total. Then, the title is characterisedas a p-dimensional vector, with each measurementrepresentativea specific doctor title. Each specific doctor should only have one label at a time [14].

Thus, there is only one rate in the vector set to 1 , with all of the others being set to 0 . Thus, if there are $p$ diverse classification features, then there should be $p \quad i=1$ ci proportions in the feature vector. (ii) Numeric structures include evidence such as the number of conference options, the number of 'likes' from partners, the number of followers, and the quantity of fans in a doctor's social media. The value of numeric structures can be directly characterised in the feature vector. If there are $q$ diverse numeric features, then there should be a q-dimensional 
vector. (iii) Textual structures include resumes and overviews. In this paper, we work the bag of words model to mine such features.

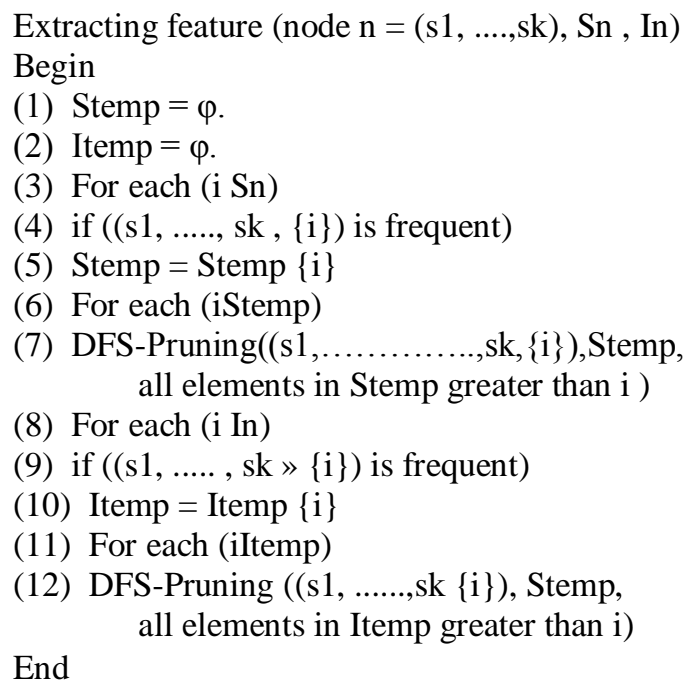

Algorithm 1: Feature extraction

Eachword is characterised as a dimension. We obtainr diverse words following word subdivision. The resume of each doctor is characterized as an r-dimensional vector. Foreach measurement, if a word appears in the doctor's resume, then the value of the conforming dimension is set to the numberof times that the word looked. Otherwise, the value of theconforming dimension is set to 0 . Succeeding the above pprocess, each doctor has a conforming $\mathrm{m}=\mathrm{i}=1$ ci $+q+$ rdimensional vector.

\section{CLUSTERING-BASED SAMPLING USING K- MEDOIDS}

Cluster sampling method is based onthe range of a label subset. In this method, we use theK-Medoidscluster. The chief concept of the cluster samplingmethod is to group all the labels in the model into K-Medoidsand select one label from each cluster. To cluster labels, weshould first produce a vector for each label. K-Medoids is clustering by segregating algorithm as like as K-means algorithm.KMedoids algorithm completes better than KMeans algorithm when the number of data points rises to maximum. It is robust in presence of clamour and outlier because medoid is less predisposed by outliers, but dispensation is more exclusive.

It act like the K-Means cluster algorithm and then it allocates each characteristics to the nearest point or node with the aid of data and also it choose the positions object also to find the needed data. The most centrally positioned instance in a cluster is measured as centroid in place of taking cruel value of the substances in K-Means clustering. This centrally located entity is called reference argument and medoid [15].

\author{
Algorithm: K-Medoids \\ Input: \\ $\mathrm{D}$, a set of d training tuples; \\ $\mathrm{k}$, the number of models in the ensemble; \\ a learning scheme \\ Output: \\ A composite model \\ Method: \\ (1) fori $=1$ to $\mathrm{k}$ do $/ /$ create $\mathrm{k}$ models: \\ (2) create bootstrap sample, Di, by sampling D with \\ replacement; \\ (3) use Di to derive a model, Mi; \\ (4) end for \\ To use the composite model on a tuple, $\mathrm{X}$ : \\ (1) if classification then \\ (2) let each of the k models classify $\mathrm{X}$ and return the \\ majority vote; \\ (3) if prediction then \\ (4) let each of the k models predict a value for $\mathrm{X}$ and \\ return the average predicted value;
}

Algorithm: K-Medoids

It minimizes the distance among centroid and data spoints means minimalize the formed error. With the help of this algorithm it finds out the secreted data.k clusters. Since obtaining a high-quality vector with tags with too few incidences using the above-mentioned method is difficult, these labels will be placed in one group prior to clustering. Because the aforesaid method only extracts supreme sample from each cluster, we only need $\mathrm{k}$ specimen experimentations. By this cluster, we established a doctor recommendation system.

\section{RECOMMENDATION SYSTEM USING MOBILE}

Our system yields recommendations for pharmacological companies and patients, and itsinternet-based whichqualifies content scrutiny and recommendations for users. This organisation offers a range of data scrutiny reports to identify, profile, update, track, and quantity the impacts of doctors. Because the outmoded approach that be dependent on on outmoded literature searches and doctor examinations is not acceptable to probable patients, our data analysis concerning ready-to-use actionable understandings and periodically updated information affords a robust podium for tracking and broadcasting the client's rendezvous through mobile technology.

\section{VIIICONCLUSION}

Sharing the information through communication technology is an essential factor for all human beings. By providing this service customer friendly service arise to elucidate this issues. CFS is a key concept for transferring 
the information. Lot of technologies were followed to share the message, however those technologies consume more computing resources and more energy. It is the essential factor for communication. This paper proposes a data mining classification method for recommendation through mobile application by using the medical dataset. To classify the user required data through mobile application we use K-NN algorithm to find out the nearest element in the labelled data and also naïve Bayesian Classifiers to find out the most likelihood data.

\section{REFERENCE}

[1] A survey of Knowledge Discovery and Data Mining process models The Knowledge Engineering Review, Vol. 21:1- 682006.

[2] Ranjita Panda, "E-waste Management: A Step towards Green Computing"

[3] DivyaTomar and Sonali Agarwal, "A survey on Data Mining approaches for Healthcare"

[4] Jesse Read, Luca Martinod,e , JaakkoHollm e, "Multi-label Methods for Prediction with Sequential Data".

[5] Nishchol Mishra, Dr.SanjaySilakari, "Predictive Analytics: A Survey, Trends, Applications, Oppurtunities\& Challenges".

[6] LI GUO , BO JIN, "Multi-Label Classification Methods for Green Computing and Application for Mobile Medical Recommendations"

[7] Bernhard Peischl, Michaela Ferk and Andreas Holziner, “ Integrating User-centred Design in an Early Stage of Mobile Medical Application Prototyping A Case Study on Data Acquistion in Health Organisations".

[8] SunitaSoni and O.P.Vyas Using Associative Classifiers for Predictive Analysis in Health Care Data Mining - International Journal of Computer Applications (0975 - 8887) Volume 4 - No.5, July 2010.

[9] PranaliDhongade, RushiLongadge and Deepak Kapgate, "A Review on Classification of Multi-label Data in Data Mining"

[10] Shubpreet Kaur and Dr. R.K.Bawa, “Future Trends of Data Mining in Predicting the Various Diseases in Medical Healthcare System"

[11] DivyaTomar and Sonali Agarwal, "A survey on Data Mining approaches for Healthcare"

[12] S. DivyaMeena and M. Revathi, "Predictive Analytics on Healthcare: A Survey"

[13] Mohamed Anis Bach Tobji, Boutheina Ben Yaghlane, and Khaled Mellouli, "A New Algorithm for Mining Frequent Itemsets from Evidential Databases”, Proceedings of IPMU'08, pp. $1535\{1542$.

[14] G. Chandrashekar and F. Sahin, "Asurvey on feature selection methods," in Comput. Electr. Eng., vol. 40, pp. 16-28, 2014.

[15] Pavel Berkhin, "A Survey of Clustering Data Mining Techniques", pp.25-71, 2002 